\title{
On Collapse of Uniform Density Sphere with Pressure
}

\section{Mahesh Chandra Durgapal $^{1}$, Pratibha Fuloria ${ }^{2}$}

${ }^{1}$ Retired Professor of Physics, Kumaun University, Naintal, India; ${ }^{2}$ Department of Physics, SSJ Campus, Kumaun University, Almora, India.

Email: garciluiz@gmail.com

Received March $1^{\text {st }}, 2010$; revised April 18 $8^{\text {th }}, 2010$; accepted May $10^{\text {th }}, 2010$.

\begin{abstract}
Adiabatic collapse solutions of uniform density sphere have been discussed by so many authors. An analysis of these solutions has been done by considering the baryonic conservation law and the no heat transfer condition. We have examined whether the pressure can remain finite or not during the collapse.
\end{abstract}

Keywords: Genral Relativity, Astrophysics, Collapse

\section{Introduction}

Radial adiabatic motion of perfect fluid spheres of uniform density, $E=E(t)$, but non-uniform pressure were discussed by Bonnor and Faulkes [1], Thompson and Whitrow [2,3] and Bondi [4] under various assumed relationships between central pressure and density. These authors discussed the problem of collapse and bounce under two assumptions: first, that the motion is isotropic or shear-free; and second, that the density is uniform. But Mishra and Shrivastava [5] showed that the condition of uniform density and regularity at the centre necessarily lead to the isotropic motion.

The theme of this paper is rather different from that of the other authors. We have examined whether the pressure can remain finite or not. We have considered the no-heat transfers (NHT) conditions (explained in the text) and baryon conservation law during the collapse. It is shown that if the fluid is isentropic or (and) the surface temperature remains constant during the collapse the pressure can not remain finite (it vanishes). On the other hand if the fluid is neither isentropic nor the surface temperature remains constant during the collapse, then the results obtained by earlier authors (Bondi, 1969) are found to be inconsistent with the baryonic conservation and NHT condition.

\section{The Metric and Uniform Density Sphere}

Vanishing shear implies that one can simultaneously introduce isotropic and co-moving coordinates

$$
\begin{gathered}
d s^{2}=y^{2} d t^{2}-R^{2}\left(d r^{2}+r^{2} d \Omega\right) \\
y=y(r, t), R=R(r, t), d \Omega^{2}=d \vartheta^{2}+\sin ^{2} \vartheta d \varphi^{2}
\end{gathered}
$$

It is assumed that the fluid's viscosity vanishes, and the adiabatic flow condition makes $T_{10}$ component of energy momentum tensor vanish in the co-moving coordinates. The energy momentum tensor can thus be written as

$$
T_{\alpha \beta}=(P+E) U_{\alpha} U_{\beta}-P g_{\alpha \beta}
$$

where $E$ and $P$ are energy density and pressure, respectively and the four-velocity,

$$
U_{\alpha} \equiv(y, 0,0,0)
$$

The hydrodynamic equations,

$T_{; \beta}^{\alpha \beta}=0$ and $U_{\alpha} T_{; \beta}^{\alpha \beta}=0$, and the equation of baryon conservation, $\left(n U^{\alpha}\right)_{; \alpha}=0 \quad$ (where $n=$ number density) give us (Misner and Sharp) [6], (Demianski) [7]

$$
\left(y^{\prime} / y\right)=-P^{\prime} /(P+E)
$$

and

$$
U^{\alpha} s,_{\alpha}=0 \text { or } s^{\circ}=0 \text { and } s^{\prime} \neq 0
$$

$\left(^{\prime}\right) \equiv$ partial differentiation w.r.t. $r ; r ;(\circ) \equiv$ partial differentiation w. r. t. t. 


\section{The Boundary Condition and Thermodynamic Relation}

For the exterior solution some authors have chosen Schwarzschild vacuum solution while others have chosen Vaidya's radiative solutions in the exterior. In the later case the heat flow is given by Kramer [8]

$$
q=-\left(K / y R^{2}\right)(T y)^{\prime}
$$

Here, $K$ is thermal conductivity. But in the cases where the exterior solution is chosen as Schwarzschild solution we get NHT conditions $(q=0)$ given by either

$(T y)^{\prime}=0$, that is, $T y=T_{b} y_{b}$ where $T_{b}=T\left(r=r_{b}\right)(6)$

$$
\text { Or } T=0 \text { (for cold stars) }
$$

$$
\text { Or } K=0
$$

The basic law of thermodynamic change is

$$
\begin{gathered}
T d s=d U+P d(1 / n) \\
n T d s=d E-h d n \text { and }(\partial E / \partial s)_{n}=n T
\end{gathered}
$$

where, $U \equiv$ specific internal energy, $s \equiv$ specific entropy and $h=(P+E) / n \equiv$ specific enthalpy. The units of $n$ are chosen so that, $P \rightarrow 0, E \rightarrow n$, and $h \rightarrow 1$.

Writing Bondi's results (1969) in the present notations, one gets

$$
\begin{gathered}
n R^{3}=B(r) \\
R=\alpha /\left(1-\beta r^{2}\right) \text { and } n R^{3}=B(r) \\
y=F R^{\circ} / R \\
R^{\circ} / R=\left(\alpha^{\circ} / \alpha\right)+\beta^{\circ} r^{2} /\left(1-\beta r^{2}\right) \\
y_{b} / y=(P+E) / E=-\frac{E^{\circ}}{3 E} \frac{R}{R^{\circ}}
\end{gathered}
$$

And

$$
P / E=\frac{\beta^{\circ}\left(r_{b}^{2}-r^{2}\right)}{\left(1-\beta r_{b}^{2}\right)\left[\alpha^{\circ}+r^{2}\left(\alpha \beta^{\circ}-\beta \alpha^{\circ}\right)\right]}
$$

$\alpha=\alpha(t), \beta=\beta(t), F=F(t), y_{b}=y\left(r=r_{b}\right), r_{b} \equiv r$ at the boundary.

Since $E=E(t)$ or $E^{\prime}=0$, we write [using Equation (10)]

$$
\begin{gathered}
\left(n^{\prime} / n^{2}\right)=-T s^{\prime} /(P+E) \\
=-\left(T y / E y_{b}\right) s^{\prime}[\text { using Equation (15)] }
\end{gathered}
$$

\section{Collapse of Uniform Density Sphere}

The collapse of uniform density sphere is discussed under various physical conditions. [We have assumed that
$A(t) \equiv$ any arbitrary function of $t$ and $B(r) \equiv$ any arbitrary function of $r$ ]

\section{4(a) using NHT condition (6a):}

Using Equation (6) in (18) one gets,

$$
\begin{gathered}
n^{\prime} / n^{2}=-\left(T_{b} / E\right) s^{\prime} \text { or } \\
(E / n)=1+T_{b}\left(s-s_{b}\right) \\
s_{b}=s\left(r=r_{b}\right) .
\end{gathered}
$$

It is obvious from Equation (19) that the entropy of an adiabatic uniform density sphere is minimum at the boundary.

4(a) (i): Isentropic case: Let the entropy be constant throughout the sphere, that is, $s=$ constant $=s_{b}$. Equation (19) gives

$$
E=n
$$

[Using Equation (11)]

$$
\begin{aligned}
E R^{3}=B(r) & R=A(t) \times B(r) \\
& R^{\circ} / R=A(t)
\end{aligned}
$$

[From Equation (13)]

$$
y=A(t) \text { or } y^{\prime}=0
$$

[From Equation (4)]

$$
P^{\prime}=0, \text { or } P=P(t)
$$

Since, $P\left(r=r_{b}\right)=0=P(t)$, the pressure vanishes within the sphere. Hence, an isentropic uniform sphere undergoes a collapse with vanishing pressure only.

4(a) (ii) Non-isentropic case with constant surface temperatures: We assume that the surface temperature remains constant during the collapse. This is very likely because there is no energy loss to the surrounding from the surface of the sphere. With $T_{b}=$ constant during the collapse one gets

$$
n=E /\left[1+T_{b}\left(s-s_{b}\right)\right]=A(t) \times B(r)
$$

[From Equation (11)]

$$
R=A(r) \times B(r)
$$

Arguments similar to those in 4(i) show that the pressure vanishes inside the sphere.

Hence, an adiabatic uniform density sphere with constant surface temperature collapses with vanishing pressure.

4(a) (iii) General case: Neither the fluid is isentropic nor the temperature of the surface remains constant. In this case

$$
E=n\left[1+T_{b}\left(s-s_{b}\right)\right]
$$

On differentiating with respect to time we obtain 


$$
\frac{E^{\circ}}{E}=\frac{n^{\circ}}{n}+\frac{T_{b}^{\circ}\left(s-s_{b}\right)}{1+T_{b}\left(s-s_{b}\right)}
$$

For an adiabatic motion the total mass energy is a constant of motion, that is,

$$
M=(4 \pi / 3) E R_{b}^{3}=\text { constant or } E^{\circ} / E=-3 R_{b}^{\circ} / R
$$

Using Equations (11), (14), (26) and (27) we get

$$
\begin{aligned}
\frac{T_{b}^{0}\left(s-s_{b}\right)}{1+T_{b}\left(s-s_{b}\right)}= & \frac{3 \beta^{\circ} r^{2}}{1-\beta r^{2}}-\frac{3 \beta^{\circ} r_{b}^{2}}{1-\beta r_{b}^{2}} \\
& =\frac{3 \beta^{\circ}\left(r^{2}-r_{b}^{2}\right)}{\left(1-\beta r^{2}\right)\left(1-\beta r_{b}^{2}\right)} \\
& =\frac{3 \beta^{\circ}\left(r^{2}-r_{b}^{2}\right)}{1-\beta\left(r^{2}+r_{b}^{2}\right)+\beta^{2} r^{2} r_{b}^{2}}
\end{aligned}
$$

No choice of functions $s=s(r), \quad \beta=\beta(t)$ and $T_{b}=$ $T_{b}(t)$ can satisfy this equation. The solutions obtained by various authors for collapsing/expanding uniform density [with Schwarzschild exterior solutions] are inconsistent with the conservation law and NHT.

4(a) (iv) Explanation of inconsistency: Equation (10) shows that $(\partial E / \partial s)_{n}=n T$, but from Equation (19) we see that $(\partial E / \partial s)_{n}=n T_{b}$. Therefore, $n T=n T_{b}$ or $T=$ $T_{b}$. Since, $T y=T_{b} y_{b} \quad$ [from Equation (6)] we get $y=$ $y_{b}=A(t)$.

Hence, $y^{\prime}=0$ or [from Equation(4)] $P^{\prime}=0 \quad \therefore P=$ $P\left(r=r_{b}\right)=0$

The pressure vanishes throughout the sphere.

\section{4(b) using NHT condition (7):}

When $T=0$ Equation (14) gives $n^{\prime}=0$ or $n=n(t)$ or, $R=A(\mathrm{t}) \times B(r) \quad$ [from Equation (9)]. As shown in 4(a) (i) the pressure vanishes inside the sphere.

\section{4(c) using NHT condition (8):}

When thermal conductivity $K=0$, it seems that all the relations of Bondi's paper are consistent. However, let us analyse this condition in some details. From Equation (10) we can see that

$$
n^{\circ} / n=E^{\circ} /(P+E) \text { and } n^{\prime} / n=E^{\prime} /(P+E)-(T / h) s^{\prime}
$$

And for $E=E(t),(T / h) s^{\prime}=-n^{\prime} / n$

When $K=0$, no heat enters or leaves any layer within the structure during the collapse that is we can consider temperature of each layer to be independent of time or $T$ $=T(r)$.

Eliminating $n$ from the twin Equations (29) we obtain (Nariai ) [9]

$$
\left(\frac{T}{h}\right)^{\circ} s^{\prime}=\frac{E^{\circ} P^{\prime}-P^{\circ} E^{\prime}}{P+E} \neq 0=\frac{E^{\circ} P^{\prime}}{P+E} \text { for } E=E(T)
$$

or

$$
n T^{\circ}=(T / h) P^{\circ}+E^{\circ}\left(P^{\prime} / s^{\prime}\right)
$$

It can be seen from Equation (16), that the right hand side of Equation (32) can not be made zero in any case.

Now, we consider a hypothetical case that during the collapse, though $K=0$, somehow the temperature of each layer changes with time making $T=T(r, t)$, but at the surface the temperature will not change with time, that is, $T_{b}^{\circ}=0$. It can be seen that

$$
E T_{b}^{\circ}=\left[E^{\circ}\left(P^{\prime} / s^{\prime}\right)+P^{\circ} T\right] r=r_{b}
$$

The right hand side of equation can not be made zero.

\section{Conclusions}

After studying adiabatic collapse of a uniform density sphere using baryon conservation law and NHT condition it is concluded that, a uniform density sphere [with Schwarzschild geometry in the exterior] always collapses adiabatically with vanishing pressure. Collapse with pressure will involve violation of either the baryonic conservation law or the no-heat flow condition. Or we can say that when the exterior geometry is defined by Schwarzschild vacuum solution then the solution given by Oppenheimer and Snyder [10] is the only valid solution for the collapse of a uniform density sphere.

\section{REFERENCES}

[1] W. B. Bonnor and M. C. Faulkes, "Exact Solutions for Oscillating Spheres in General Relativity," Monthly Notices of the Royal Astronomical Society, Vol. 137, 1967, pp. 239-251.

[2] I. H. Thompson and G. J. Whitrow, "Time-Dependent Internal Solutions for Spherically Symmetrical Bodies in General Relativity-I. Adiabatic collapse," Monthly Notices of the Royal Astronomical Society, Vol. 136, 1967, pp. 207-217.

[3] I. H. Thompson and G. J. Whitrow, "Time-dependent internal solutions for spherically symmetrical bodies in general relativity-II. Adiabatic radial motions of uniformly dense spheres," Monthly Notices of the Royal Astronomical Society, Vol. 139, 1968, pp. 499-513.

[4] H. Bondi, "Gravitational Bounce in General Relativity," Monthly Notices of the Royal Astronomical Society, Vol. 142, 1969, pp. 333-353.

[5] R. M. Misra and D. C. Srivastava, "Relativity-Bounce of Fluid Spheres," Nature Physical Science, Vol. 238, 1972, p. 116.

[6] C. W. Misner and D. H. Sharp,"Relativistic Equations for Adiabatic, Spherically Symmetric Gravitational Collapse," Physical Review B, Vol. 136, No. 2B, 1964, pp. 
B571-576.

[7] M. Demianski, "Relativistic Astrophysics," Pergamon Press, New York, 1985.

[8] D. Kramer, "Spherically Symmetric Radiating Solution with Heat Flow in General Relativity," Journal of Mathematical Physics, Vol. 33, No. 4, 1992, pp. 14581462.
[9] H. Nariai, "A Simple Model for Gravitational Collapse with Pressure Gradient," Progress of Theoretical physics, Vol. 38, No. 1, 1967, pp. 92-106.

[10] J. R. Oppenheimer and H. Snyder, "On Continued Gravitational Contraction," Physical Review, Vol. 56, No. 5, 1939, pp. 455-459. 